\title{
Targeted Delivery of siRNA through Nanocomplex using Specific Fusion Peptides for Breast Cancer Treatment
}

\author{
Jang Hyuk Bang ${ }^{1}$, Kyung Ah Kim ${ }^{1}$, Yeong Chae Ryu ${ }^{1}$, Byoung Choul Kim ${ }^{1}$, and \\ BYEONG HEE HWANG ${ }^{1}$
}

${ }^{1}$ Incheon National University

November 17, 2021

\begin{abstract}
Breast cancer is one of the serious diseases and has the second-highest mortality in women worldwide. RNA interference has been developed as a promising way of specific cancer treatment by silencing oncogenes efficiently. However, small RNAs exhibits difficulties in specific cellular uptake and instability. Therefore, we designed novel fusion peptides (RS and RT) for an efficient, stable, and specific delivery of small RNAs. Both RS and RT peptides could form self-assembled nanocomplexes via electrostatic attraction. RS nanocomplexes exhibited prolonged stability, enhanced cellular uptake, and target gene silencing by siRNAs to MDA-MB-231 breast cancer cells. Moreover, RS nanocomplexes successfully inhibited breast cancer cell growth via specific and efficient siRNA delivery. Furthermore, in vitro and in vivo safety tests showed negligible cytotoxicity and neither tissue damage nor significant inflammatory cytokine release. Therefore, the RS nanocomplexes could be expected to become a promising siRNA delivery platform for the treatment of breast cancer or other cancers.
\end{abstract}

\section{Introduction}

Breast cancer is the most common type of cancer, and at the same time, it has the second-highest mortality rate in women worldwide. Statistically, breast cancer affects about 100 out of 100,000 women in the United States each year, resulting in a total of more than 230,000 new patients. ${ }^{[1]}$ Currently, various treatments, such as chemotherapy and hormonal therapy, are being used to treat breast cancer or prevent a recurrence. Still, they exhibit drug resistance and can cause non-specific cell damage. ${ }^{[2,3]}$ Furthermore, chemotherapy's side effects include fatigue, diarrhea, constipation. ${ }^{[4]}$ In addition, hormonal therapy is limited because it cannot be used in about $30 \%$ of women. ${ }^{[5]}$ Recently, treatment methods that alleviate systemic toxicity are being studied in chemotherapy and hormonal therapy. However, there are still reports of side effects such as nausea, mouth ulcers, and mild cognitive impairment, continuing concerns about toxicity. ${ }^{[6]}$ For these reasons, it is necessary to develop a new anticancer drug that is effective without side effects.

siRNAs are double-stranded synthetic RNAs composed of 21-23 base pairs and can become a component of the RISC complex, which could cut the desired mRNA sequence in the cells. ${ }^{[7,8]}$ Due to specific gene silencing and minimal side effects, siRNAs are currently being studied to treat various diseases and effectively kill cancer cells by knocking down the expression of oncogenes. ${ }^{[9]}$ The FDA has approved three siRNA drugs of patisiran in 2018, givosiran in 2019, and lumasiran in 2020. ${ }^{[10-12]}$ However, siRNAs are difficult to deliver into cells due to their strong negative charge and are easily degraded by nucleases. Therefore, an effective and stable delivery technique is required for siRNA delivery.

siRNA delivery methods are majorly divided into physical and chemical methods. First, physical methods included electroporation, iontophoresis, sonophoresis, magnetofection, gene gun, and laser beam transduction. They showed high delivery efficiency of nucleic acids. However, special equipment is required. ${ }^{[13]}$ Second, chemical methods include polymer-based, liposome-based, and peptide-mediated carriers. They have been 
recognized for their delivery efficiency, but polymer-based carriers presented clinical difficulties due to their high toxicity. ${ }^{[14,15]}$ Also, liposome-based carriers showed low toxicity, instability, drug leakage, and difficult sterilization due to sensitivity to high temperature. ${ }^{[16,17]}$

Peptide-mediated carriers have been studied since cell-penetrating peptides were first discovered using phage display in 1988. ${ }^{[18,19]}$ Nowadays, cell-penetrating peptides (CPP) are widely used due to their advantages of excellent penetrating ability, easy conjugation, imparting additional functions depending on the amino acid sequence, and relatively low cytotoxicity. ${ }^{[20]}$ The most frequently used CPPs include transportan, polyarginine, antennapedia, and TAT. Among them, polyarginine, an analog of TAT, could penetrate the cellular membrane most efficiently with relatively low toxicity. ${ }^{[21]}$ Furthermore, because it has a positive charge, it neutralizes the negative charge of nucleic acids and facilitates intracellular transfer through hydrogen bonding with the cell membrane. ${ }^{[22-25]}$ However, polyarginine-based carriers cannot deliver drugs targeting certain cell types. So, the non-targeted delivery of cytotoxic drugs can cause various side effects, i.e., damage to normal tissues. Therefore, a new targeted delivery method needs to be developed to maximize the therapeutic efficacy and minimize side effects.

Homing peptides can become a potent ligand for specific delivery due to the affinity to particular proteins on the cell membrane surface. Among them, TT1 and SP82 homing peptides were reported as specific to breast cancer cells. TT1 peptide was screened through the M13 phage display technique using the peptide library of cyclic nine amino acids. ${ }^{[26]}$ The TT1 was revealed to bind the p32 receptor on breast cancer cells' surfaces. Next, protease could cut the front part of the c-terminus serine of the peptide, leaving a C-end Rule (or CendR) motif. Then, the cleaved TT1 could be delivered into the cells through the NRP-1 receptor-mediated endocytosis. And, the SP82 peptide was screened specifically to breast cancer cells through the M13 phage display technique. In addition, the SP82 endocytosis mechanism still needs to be revealed. ${ }^{[27]}$ Furthermore, these homing peptides need a conjugation strategy to use for the specific delivery.

This study developed the nanocomplex-mediated siRNA delivery based on novel fusion peptides targeting breast cancer cells (Fig. 1). The homing peptide (SP82 and TT1) was conjugated to oligoarginine $\left(\mathrm{R}_{11}\right)$ with a linker (GGGG), respectively (Additional file 1: Table S1). The oligoarginine with a positive charge could spontaneously form nanocomplexes (NCs) with siRNAs with electrostatic attraction. The formed NCs were characterized in size, surface charge, and stability. The delivery efficiency and specificity of NCs were quantitatively analyzed using flow cytometry. The cellular internalization of NCs was confirmed using fluorescence z-stack imaging. The efficacy of delivered siRNA was confirmed using mRNA gene silencing and viability test of breast cancer cells. Finally, the biocompatibility of RS-NC was assessed by histological analysis and cytokine ELISA of intradermally injected mouse skin.

\section{Materials and Methods}

\subsection{Materials}

The cancer growth-inhibiting (CGI) siRNA and other siRNAs were synthesized by Genolution Pharmaceuticals Inc. (Table S1). (Seoul, Korea). The Cy3-labeled siRNA was synthesized by GenePharma Co., Ltd. (Shanghai, China). The FITC-labeled siRNA was synthesized by Bioneer Co. (Daejeon, South Korea). $\mathrm{R}_{11}$ single peptide and RS and RT fusion peptides in Table S2 were synthesized by GL Biochem, Ltd. (Shanghai, China) with more than 95\% purity. Dulbecco's phosphate-buffered saline (DPBS) and heparin sodium salt (from porcine intestinal mucosa) were purchased from Sigma-Aldrich (MO, USA). Agarose was purchased from Becton Dickinson and Company (Cockeysville, MD). 10,000× TopRed Nucleic Acid Gel Stain was purchased from GenomicBase (Seoul, South Korea). Tris(hydroxymethyl) aminomethane (Tris) was purchased from Glentham Life Sciences Ltd. (Corsham, UK). Acetic acid (glacial) was purchased from Merck (Darmstadt, Germany). 6× DNA loading dye was procured from Biofact Co., Ltd. (Daejeon, South Korea). For cell cultures, Dulbecco's modified eagle's medium (DMEM; Corning, MA, USA), fetal bovine serum (FBS; PAN Biotech, Bavaria, Germany), and penicillin-streptomycin (Life Technologies, CA, USA) were procured. Opti-MEM and $0.25 \%$ trypsin-EDTA (1x) were purchased from Thermo Fisher Scientific, Inc. (MA, USA). Hoechst 33342 was purchased from Invitrogen, Inc. (CA, USA). Phalloidin was procured from 
BioActs, Inc. (Incheon, South Korea). Nuclease-free water (NFW) was purchased from Integrated DNA Technologies, Inc (IA, USA). All cell culture flasks and plates were purchased from NEST Biotechnology Co., Ltd (Wuxi, China). CCK cell viability assay kit was procured from Donginbio, Inc. (Seoul, Korea). Tri-RNA solution was purchased from Biotech Co. (Kaohsiung, Taiwan)

\section{$2.2 \mathrm{NC}$ formation}

$20 \mu \mathrm{M}$ of siRNA, $100 \mu \mathrm{M}$ FITC-siRNA, or $100 \mu \mathrm{M}$ Cy3-siRNA were diluted in NFW, and $600 \mu \mathrm{M}$ peptides were dissolved in DPBS. All solutions were stored at $-80 \mathrm{oC}$ until use. The fixed amount of siRNA was incubated with a fusion peptides solution of a nitrogen/phosphate ratio to proper volume filled with DPBS as described below, at room temperature for 30 minutes. The nitrogen/phosphate $(\mathrm{N} / \mathrm{P})$ ratio was calculated from nitrogens of fusion peptides divided by phosphates of siRNAs. First, the formation of NCs was observed using gel retardation assay. $10 \mathrm{pmol}$ of siRNA was incubated with RS peptide of $\mathrm{N} / \mathrm{P}$ ratio $(0.005,0.01$, $0.025,0.05,0.1,0.25,0.5,1,3$, and 5$)$ and $\mathrm{RT}$ peptide of $\mathrm{N} / \mathrm{P}$ ratio $(1,5,10,20,30,40,50,60$, and 70$)$ in final volume of $10 \mu \mathrm{L}$ filled with DPBS as described above. After the addition of $6 \times$ loading dye, mixed solutions were loaded to wells of $1 \%(\mathrm{w} / \mathrm{v})$ agarose gel in $1 \times$ TAE buffer $(40 \mathrm{mM}$ tris, $20 \mathrm{mM}$ acetic acid, $1 \mathrm{mM}$ EDTA, $\mathrm{pH}=8.6$ ). Electrophoresis was run at $100 \mathrm{~V}$ for 30 minutes using the Mupid-2plus electrophoresis system (Optima Inc., Tokyo, Japan). TopRed stained RNA bands were visualized using the ChemiDoc XRS + System (Bio-Rad, CA, USA).

\subsection{Size and zeta potential of NCs}

The size and zeta potential of the NCs were measured by a zetasizer. NCs with 200 pmol of siRNA were formed at $\mathrm{N} / \mathrm{P}$ ratio 1,3 , and 5 of RS peptide and $\mathrm{N} / \mathrm{P}$ ratio 5,20 , and 30 of RT peptide at room temperature for 30 minutes. Three N/P ratios of each peptide were selected to observe different aspects of NCs. Formed NCs were diluted to $200 \mathrm{nM}$ in $1 \mathrm{ml}$ DPBS and filtered with a $0.45-\mu \mathrm{m}$ syringe filter (GVS, Bologna, Italy). Each NC was loaded in a cell (Ratiolab, Dreieich, Germany). Hydrodynamic radius and zeta potential were measured using a zetasizer (Malvern Panalytical, Ltd., Malvern, UK, $\mathrm{n}=3$ ).

\subsection{Stability test of NCs}

The NCs were counted to confirm stability. First, 50 pmol of siRNA and each peptide $\left(\mathrm{R}_{11}, \mathrm{RS}\right.$, RT) were mixed to form NCs in a total volume of $120 \mu \mathrm{L}$ in DPBS as described above. After 30 minutes of incubation, $20 \mu \mathrm{L}$ of each NC was loaded in a slide (Arthur Slide; NanoEnTek, Seoul, Korea), and images were obtained using a fluorescence cell counter (Arthur; NanoEnTek, Seoul, Korea). The number of each particle was calculated by the imaging program Image J (NIH, USA). ${ }^{[28]}$

\subsection{Fluorescence imaging of NCs}

Cellular uptake of NCs was observed using fluorescence imaging. First, $1 \times 10^{4}$ MDA-MB-231 cells were seeded to wells of a 24-well plate and incubated for 16 hours in standard conditions. $200 \mathrm{nM}$ Cy3-labeled siRNAs were incubated with $\mathrm{R}_{11}$, RS, and RT peptides at the N/P ratio 20, 5 , and 30 in $200 \mu \mathrm{L}$ were treated into a total volume of $500 \mu \mathrm{L}$ and incubated at room temperature for 30 minutes, respectively. At 4 hours after NC addition, the cells were rinsed three times with DPBS $200 \mu \mathrm{L}$, and the cells were fixed by $200 \mu \mathrm{L}$ of $10 \%$ formalin solution for 10 minutes. Next, the cells were added by $200 \mu \mathrm{L}$ of $0.1 \%$ Triton X-100 in PBS (0.1\% PBST). After a 10-minute incubation, cells were added by $200 \mu \mathrm{L}$ of $2 \%$ (w/v) BSA in $0.1 \%$ PBST and incubated at room temperature for 30 minutes. Then, the cells were incubated in the Hoechst 33342 $(10 \mathrm{mg} / \mathrm{ml}$ in PBS) for 10 minutes. After rinsing cells with $200 \mu \mathrm{L}$ of PBS twice, the cells were incubated in the Phalloidin (diluted by PBS-T as 1:200 v/v) at room temperature for one hour. After washing twice with $200 \mu \mathrm{L}$ of DPBS, the cells were observed at $150 \times$ magnification by a fluorescence microscope (Ti-E; Nikon, Tokyo, Japan).

\subsection{Flow cytometry for cellular uptake analysis}

NC-mediated cellular uptake efficiency was analyzed using flow cytometry. MDA-MB-231, MCF-7, BT-474, SK-BR-3 cells were used for breast cancer cell types, and RAW264.7 cell was used for a normal cell type. 
First, $2.5 \times 10^{4}$ cells were seeded to a well of a 6 -well plate and incubated at $37 \mathrm{oC}$ for 16 hours in a $5 \% \mathrm{CO}_{2}$ incubator. Then, $200 \mathrm{nM}$ FITC-labeled siRNA was incubated with $\mathrm{R}_{11}$, RS, RT peptides of N/P ratio 20, 5,30 in $2 \mathrm{~mL}$ of total volume at room temperature for 30 minutes. Next, at 4 hours at $37^{\circ} \mathrm{C}$ and $5 \% \mathrm{CO}_{2}$ after NC addition, cells were rinsed three times with DPBS $1 \mathrm{~mL}$ and detached using $0.25 \%$ Trypsin $1 \mathrm{~mL}$ for 2 minutes. After $1 \mathrm{~mL}$ DMEM addition, cells were centrifuged at $300 \times \mathrm{g}$ for 5 minutes. Finally, dispersed cells were loaded to the flow cytometry (Cytoflex; Beckman Coulter, MA, USA) for analysis.

\subsection{Z-stack imaging of RS-NC}

The intracellular localization of RS-NC was observed by z-stack imaging. First, $2.0 \times 10^{5}$ MDA-MB-231 cells were seeded to a 35-mm confocal dish (SPL Life Sciences Co., Ltd., Pocheon, South Korea) and incubated overnight in standard conditions. Next, the $200 \mathrm{nM}$ FITC-labeled siRNA was incubated with RS peptide $(\mathrm{N} / \mathrm{P}$ ratio 5) for 30 minutes at RT. After four-hour incubation with NC, the actin filaments of cells were stained Phalloidin (diluted by PBS-T as 1:200 v/v). The bright-field, fluorescence, and z-stack images were obtained at $900 \times$ magnification using a fluorescence confocal microscope (Ti2; Nikon, Tokyo, Japan). The image was analyzed at the single-molecule level using super-resolution radial fluctuation (SRRF). ImageJ software was used to merge the fluorescence images of FITC and Phalloidin. ${ }^{[28]}$

\subsection{Cell viability inhibited by NC-mediated siRNA delivery}

The inhibition by NC-mediated siRNA delivery was optimized depending on the N/P ratio and siRNA concentration using the viability assay of breast cancer cells. First, MDA-MB-231 breast cancer cells of 5.0 $\times 10^{3}$ were seeded to wells of a 96 -well plate and incubated at $37 \mathrm{oC}$ for 16 hours in a $5 \% \mathrm{CO}_{2}$ incubator (Esco Micro Pte. Ltd., Changi, Singapore). For N/P ratio optimization, 10 pmol of CGI siRNA were mixed with the N/P ratio of $1,2.5,5,7.5,10$ for RS peptide and 10,20,30,40,50 for RT peptide in $100 \mu \mathrm{L}$ of total volume as described above. For siRNA concentration, 100, 200, 300, and 400 pmol of CGI siRNA was mixed with RS peptide of N/P ratio 5 of and $R_{11}$ peptide of N/P ratio 20. Then, $100 \mu \mathrm{L}$ of each solution was added to each well and incubated for 24 hours at $37 \mathrm{oC}$ in a $5 \% \mathrm{CO}_{2}$ incubator. The same procedure was repeated. $10 \mu \mathrm{L}$ of CCK solution was added to each well. After incubation for 2 hours, the absorbance at $450 \mathrm{~nm}$ was measured by the Epoch2 microplate spectrophotometer (BioTek Instruments, Inc., VT, USA)

\subsection{Real-time PCR}

RS-NC-mediated gene silencing was measured using real-time PCR of cDNA. First, $1.0 \times 10^{5}$ MDA-MB-231 cells were seeded to wells of a 6 -well plate and incubated for 16 hours at standard conditions. Then, 200 nM Kaiso siRNA was incubated with RS peptide of N/P ratio 5 in $2 \mathrm{~mL}$ of total volume with Opti-MEM at room temperature for 30 minutes. After four-hour incubation with RS peptide, the buffer was changed by DMEM and incubated for 20 hours at standard conditions. Next, cells were rinsed with NFW three times, and the total RNA of the cells was purified by following the manufacturer's protocol of Tri-RNA solution (Biotech Co., Kaohsiung, Taiwan). Absorbances at 260 and $280 \mathrm{~nm}$ of purified RNA were measured using the Take3 micro-volume plate and Epoch2 microplate spectrophotometer. Then, according to the manufacturer's protocol, cDNA of total RNA $100 \mathrm{ng}$ was synthesized using the ReverTra Ace@ $\mathrm{R}$ qCR RT Master Mix and gDNA Remover kit (Promega, WI, USA). The qPCR reaction of synthesized cDNA was

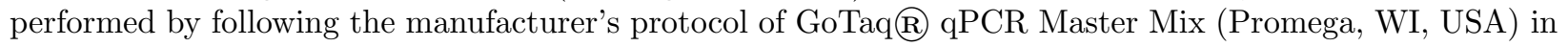
the QuantStudio 3 real-time PCR system (Applied Biosystems, CA, USA). The thermal cycle was composed of pre-denaturation at $95^{\circ} \mathrm{C}$ for 60 seconds, denaturation at $95^{\circ} \mathrm{C}$ for 15 seconds, annealing at $55^{\circ} \mathrm{C}(\beta$-actin), $53.2^{\circ} \mathrm{C}$ (KAISO), $52.4^{\circ} \mathrm{C}$ (LIPIN1), $51.2^{\circ} \mathrm{C}(\mathrm{PICH}), 58.9^{\circ} \mathrm{C}(\mathrm{PML})$ for 30 seconds, and extension at $72^{\circ} \mathrm{C}$ for 60 seconds. The relative expression of each mRNA was calculated using the $\Delta \Delta \mathrm{Ct}$ method and normalized by the mRNA expression level of $\beta$-actin.

\subsection{Endocytosis pathway of RS-NC}

The endocytosis pathway of the RS-NC was revealed using flow cytometry and endocytosis inhibitors. First, $2.0 \times 10^{5}$ MDA-MB-231 cells were seeded to wells of a 6 -well plate. After 18-hour incubation at standard conditions, $10 \mu \mathrm{g} / \mathrm{mL}$ of chlorpromazine, $5 \mathrm{mM}$ of Methyl- $\beta$-cyclodextrin, $1 \mu \mathrm{M}$ of Cytochalasin $\mathrm{D}$, and 1 
$\mu \mathrm{g} / \mathrm{mL}$ Filipin III were added to each well, respectively, and incubated for 30 minutes. Then, RS-NC (N/P ratio 5) with $200 \mathrm{nM}$ FITC-labeled siRNA was added to each well and incubated for 4 hours at standard conditions. After rinsing cells with DPBS three times, cells were detached using $0.25 \%$ Trypsin $200 \mu \mathrm{L}$ for 2 minutes. After $1 \mathrm{~mL}$ DMEM addition, cells were centrifuged at $300 \times \mathrm{g}$ for 5 minutes. Finally, dispersed cells were loaded to the flow cytometry for analysis.

\subsection{Cytocompatiblity of fusion peptides}

The cytocompatibility of fusion peptides was assessed by CCK-8 cell viability assay. MDA-MB-231 and HDF-n cells were used as breast cancer and normal cells, respectively. First, $5.0 \times 10^{3}$ MDA-MB-231 and HDF-n cells were seeded to wells of a 96-well plate and incubated for 16 hours at standard conditions. Next, 1, 2, 3, $4 \mu \mathrm{M}$ RS peptide solutions and 10, 15, 20, $25 \mu \mathrm{M}$ RT peptide solutions were added to each well. After 48-hour incubation at standard conditions, $10 \mu \mathrm{L}$ of CCK solution was added to each well. After incubation for 2 hours, the absorbance at $450 \mathrm{~nm}$ was measured by the Epoch2 microplate spectrophotometer.

\subsection{Histological analysis of skin tissue}

The RS-NC-injected mouse skin tissue was observed by histological analysis after H\&E staining. All animal experiments were performed according to the protocol approved by the Institutional Animal Care and Use Committee (IACUC) of Incheon National University (INU-ANIM-2021-01). First, RS-NC (N/P ratio 5) containing $200 \mathrm{pmol}$ of negative control siRNA were incubated for 30 minutes at RT. $30 \mu \mathrm{L}$ of RS-NC and DPBS as negative control were injected intradermally to epilated back of each five-week-old BALB/c mouse (Orientbio, Inc., Seongnam, South Korea). After 4 days, the injected skin tissues of the euthanized mice were excised and fixed in 10\% (w/v) formalin (Sigma-Aldrich, MO, USA). After 22 hours, the tissue samples were dehydrated, paraffin-embedded by embedding center (Histostar; Thermo Fisher Scientific, MA, USA), and finally sectioned to $4 \mu \mathrm{m}$ thickness by microtome (HM 355S; Thermo Fisher Scientific, MA, USA), and placed on the slide. After deparaffinization, the tissue samples were stained with hematoxylin and eosin (BioGnost, Zagreb, Croatia). Then, the slides were observed with Leica DM1000 microscope (Leica Microsystems, Hesse, Germany) under 20× magnification.

\subsection{ELISA assay of inflammatory biomarkers}

Levels of proinflammatory cytokines caused by RS-NC were measured using IL-6 and TNF-alpha ELISA assay. First, RS-NC (N/P ratio 5) containing 200 pmol of negative control siRNA were incubated for 30 minutes at RT. Then, $30 \mu \mathrm{L}$ of RS-NC and DPBS as negative control were injected intradermally to three spots on epilated back of each five-week-old BALB/c mouse (Orientbio, Inc., Seongnam, South Korea). After three days, the skin tissue of euthanized mouse was excised, and skin samples were sectioned using a $5 \mathrm{~mm}$ biopsy punch (Kai Medical, Tokyo, Japan). Then, sectioned samples in lysis buffer (RayBiotech, Georgia, USA) were cut into small pieces using scissors (EpoS Medical, Tuttlingen, Germany) for one minute. Next, samples were homogenized using a homogenizer (T10BASIC, IKA, Staufen, Germany) at 8,000 rpm for 1 minute. ELISA assays were conducted using mouse IL-6 ELISA kit and mouse TNF-alpha ELISA kit (RayBiotech, Georgia, USA). Cytokines' standard curves were obtained using four logistic parameters. Finally, cytokine levels were calculated by substituting absorbance values into the equation of a trend line.

\subsection{Statistical analysis}

The quantitative data were presented as mean \pm standard deviation. The statistical significance of the differences was evaluated using a $p$-value less than $0.05,0.01$, and 0.001 calculated by a t-test.

\section{Results}

\subsection{Formation, characteristics, and stability of NCs}

Formation, characteristics, and stability of NCs were examined using novel RS and RT fusion peptides (Fig. 2A-D). First, NC formation was checked using the electrophoretic mobility shift assay. RS peptides started to retard siRNA bands at a $0.5 \mathrm{~N} / \mathrm{P}$ ratio and fully retarded them at three and above $\mathrm{N} / \mathrm{P}$ ratios (left picture 
of Fig. 2A). On the other hand, RT peptides started to retard siRNA bands at a $1 \mathrm{~N} / \mathrm{P}$ ratio, however, do not fully retarded until a $70 \mathrm{~N} / \mathrm{P}$ ratio (right picture of Fig. $2 \mathrm{~A}$ ).

Second, RS-NCs had a hydrodynamic radius of 128.6, 467.0, and $278.5 \mathrm{~nm}$, polydispersity index of 0.17 , 0.35 , and 0.29 , and zeta potential of $-23.2,3.1$, and $10.7 \mathrm{mV}$ at the $\mathrm{N} / \mathrm{P}$ ratios of 1,3 , and 5 (Figs. $2 \mathrm{~B}$ and S1A). Furthermore, RT-NCs had a hydrodynamic radius of $340.3,235.7$, and $213.4 \mathrm{~nm}$, polydispersity index of $0.43,0.24$, and 0.24 , and zeta potential of $5.0,9.2$, and $12.6 \mathrm{mV}$ at the $\mathrm{N} / \mathrm{P}$ ratios of 5,20 , and 30 (Figs. $2 \mathrm{C}$ and $\mathrm{S} 1 \mathrm{~B})$.

Third, for stability test using fluorescence particle count, RS-NCs remained over $90 \%$ until 24 hours and over $60 \%$ until 72 hours in the $10 \%$ serum condition (Figs. 2D and S2, Table S3). On the other hand, RTNCs remained below $40 \%$ until 4 hours and completely disappeared at 48 hours. For comparison, $\mathrm{R}_{11}$-NCs, positive control, remained below $10 \%$ until 4 hours and completely disappeared at 24 hours.

\subsection{Cellular uptake and internalization of NCs}

Cellular uptake efficiency via $\mathrm{R}_{11^{-}}$, RS- and RT-NCs was checked using the flow cytometry and $200 \mathrm{nM}$ Cy3labeled siRNAs (Fig. 3). Red and green populations represented cell counts of free siRNA and NC-mediated delivery, respectively. RS-NCs showed fluorescence-positive populations of 93.0, 81.5, 55.1, 32.5, and 21.3\% in MDA-MB-231, BT-474, MCF-7, SK-BR-3, and RAW 264.7 cells. On the other hand, RT-NCs showed fluorescence-positive populations of 6.5, 4.6, 4.6, and 0.6\% in MCF-7, BT-474, MDA-MB-231, and SK-BR-3 cells. RT-NCs were not applied to RAW 264.7 cells due to low cellular uptake efficiency. For comparison, $\mathrm{R}_{11}$-NCs showed fluorescence-positive populations of $20.3,3.7,2.0,1.0$, and $0.8 \%$ in MCF-7, BT-474, MDAMB-231, RAW264.7, and SK-BR-3 cells in high order. Resultantly, RS-NCs showed the best cellular uptake efficiency. Therefore, RS-NC was chosen for further studies.

MDA-MB-231 cellular uptake and internalization of NCs were observed using fluorescence microscopy and Z-stack imaging (Fig. 4). Blue, green, and red fluorescences represented the nucleus (Hoechst 33342), actin filament (Phalloidin), and NCs (Cy3), respectively (Fig. 4A). RS-NCs are represented as well-dispersed red dots (NCs) in the $\mathrm{Cy} 3$ image, and some of the red dots entered into cells, and some of them remained outside of cells in the merged image. RT-NCs are represented as some red dots in the Cy3 image, and most red dots are located close to the cells in the merged image. $\mathrm{R}_{11}$-NCs showed smaller dots than RT-NCs in the Cy3 image, and most red dots are located close to the cells in the merged image. On the other hand, only siRNAs did not show any red dots in the Cy3 image. Next, the z-stack image indicated that RS-NCs are located in MDA-MB-231 cells (Fig. 4B). Red and green fluorescence represented actin filament (Cy3) and RS-NC (FITC). The z-stack cross-section images of the $\mathrm{x}$ and $\mathrm{y}$-axis white dotted line indicated RS-NCs are located inside the cell.

The endocytosis pathway of RS-NCs into MDA-MB-231 cells was studied using flow cytometry and endocytosis inhibitors (Fig. 5). Red and green populations represented cell counts without endocytosis inhibitor and with endocytosis inhibitor, respectively. As a result, chlorpromazine, methyl- $\beta$-cyclodextrin, cytochalasin D, and filipin III inhibited RS-NC endocytosis of $27 \%, 35 \%, 23 \%$, and $19 \%$, respectively. In addition, chlorpromazine, methyl- $\beta$-cyclodextrin, cytochalasin D, and filipin III were known inhibiting clathrin-mediated endocytosis, lipid raft-mediated endocytosis, caveolin-mediated endocytosis, and micropinocytosis, respectively.

\subsection{Cell viability reduced by RS-NC-mediated siRNA delivery}

The cancer growth-inhibiting (CGI) siRNAs were delivered via RS-NCs into MDA-MB-231 breast cancer cells. Reduced cell viabilities were measured using the CCK-8 assay (Figs. 6 and S4). First, the N/P ratios of RS-NCs were optimized for reducing cancer cell viability (Fig. 6A-C). In Fig. 6A, the CGI siRNA reduced $22 \%, 24 \%$, and $24 \%$ of cancer cell viability when $\mathrm{N} / \mathrm{P}$ ratios are 5.7 .5 , and 10 with statistical significance $\left(p\right.$-values: $1.1 \times 10^{-8}, 2.3 \times 10^{-3}$, and $\left.6.9 \times 10^{-5}\right)$. N/P ratio of 5 was chosen for the following experiments. In Fig. 6B, Kaiso siRNA reduced $15 \%$ of cancer cell viability when the N/P ratio was 10 with statistical significance ( $p$-values: $\left.1.5 \times 10^{-3}\right)$. On the other hand, in Fig. $6 \mathrm{C}$, PICH siRNA inhibited $9 \%$ and $15 \%$ of 
cancer cell viability when the N/P ratio was 1 and 10 with statistical significance $\left(p\right.$-values: $2.1 \times 10^{-2}$ and $\left.1.2 \times 10^{-3}\right)$.

Second, cell viability was measured under various CGI siRNA concentrations via RS-NC-mediated delivery (Fig. 6D). The 100, 200, and $400 \mathrm{nM}$ CGI siRNAs via RS-NC reduced 17\%, 22\%, and $36 \%$ of cancer cell viability with statistical significance ( $p$-values: $1.2 \times 10^{-3}, 6.5 \times 10^{-6}$ and $\left.3.2 \times 10^{-6}\right)$. Third, mRNA expression was quantified using quantitative polymerase chain reaction when Kaiso and PICH siRNAs were delivered to MDA-MB-231 cells via NCs (Fig. 6E). Resultantly, RS- and $\mathrm{R}_{11}$-NCs reduced $56 \%$ and $13 \%$ of

Kaiso mRNA expression compared to the non-treated group. RS- and $\mathrm{R}_{11}$-NCs reduced $22 \%$ and $11 \%$ of PICH mRNA expression compared to the non-treated group. RS-NCs reduced statistically significant Kaiso and PICH mRNA expression compared to $\mathrm{R}_{11}$-NCs $\left(p\right.$-value $=1.9 \times 10^{-5}$ and $\left.4.0 \times 10^{-3}\right)$.

\subsection{Biocompatibility of peptides and RS-NC}

Cytocompatibility of RS and RT peptides was assessed using the CCK-8 assay of MDA-MB-231 and HDFn cells (Fig. 7). First, the RS peptide did not show any significant decrease of cell viability in all peptide concentrations for both cells. Also, the RT peptide did not represent a statistically significant decrease of cell viability in most peptide concentrations except $15 \mu \mathrm{M}$ for MDA-MB-231 cells $(p$-value $=0.032)$.

Biocompatibility of RS-NC was tested by histological analysis and inflammatory cytokine levels of RSNC-injected Balb/c mouse's back skin intradermally (Fig. 8). First, skins injected by PBS and RS-NCs with negative control siRNAs were compared using H\&E-stained histological analysis (Fig. 8a). As a result, H\&E-stained both skins maintained similar thickness of dermis layer and did not represent any morphological difference. Moreover, no tissue damage or inflammatory infiltration was observed. Second, TNF- $\alpha$ and IL-6 levels of solubilized skin tissue were measured by ELISA analysis (Fig. 8b). Both cytokine levels did not represent a statistically significant difference between PBS and RS-NCs with negative control siRNAs. In summary, RS peptide and RS-NC were confirmed as cytocompatible and biocompatible.

\section{Discussion}

This research has developed an NC-mediated targeted siRNA delivery method using novel RS and RT fusion peptides (Fig. 1). First, RS and RT fusion peptides were composed of polyarginine peptide interacting siRNAs, a linker, and the SP82 and TT1 peptides targeting breast cancer cells in series, respectively. Then, RS and RT peptides electrostatically attracted siRNAs and formed self-assembled NCs (Fig. 2). Especially, RS-NCs penetrated breast cancer cells selectively and highly efficiently (Fig. 3\&4). In addition, RS-NCdelivered siRNAs effectively inhibited breast cancer cells (Fig. 6). These results implied the synergistic effect of NC-forming and membrane-penetrating abilities of poly-arginine and selectively binding to breast cancer cells of SP82 peptide. Moreover, improved penetration could be explained by the combinatorial effect of proper size, evenness, weakly positive surface charge, high stability, and high biocompatibility of the RS-NC (Fig. 3).

The different cell-penetrating efficiencies between RS- and RT-NCs could be caused by the inherent property of SP82 and TT1 peptides and the difference of formed NCs. First, the SP82 peptide had more hydrophobicity than the TT1 peptide. The RS peptide had phenylalanine, glycine, alanine, isoleucine, and three leucines. On the other hand, the RT peptide consists of glycine and three alanines. Therefore, the hydrophobicity of RS peptides could form NC more compactly after charge neutralization. It was also reported that peptides' hydrophobicity could increase cell penetration efficiencies. ${ }^{[22]}$ Moreover, the predicted alpha-helical structure of RS peptide and hydrophobicity could help to interact with membrane and escape from endosome (Figs. S5 and S6). ${ }^{[23]}$

Furthermore, the hydrophobicity of RS peptides also affected the size and evenness of NCs when an electrical charge was neutralized. For example, the size and PdI of RS-NC increased and decreased as the N/P ratio increased. On the other hand, the size and PdI of RT-NCs decreased as the N/P ratio increased. This result indicated that electrical attraction is major interaction when the $\mathrm{N} / \mathrm{P}$ ratio is low. However, as the $\mathrm{N} / \mathrm{P}$ ratio increases, hydrophobic interaction between peptides or siRNAs becomes more significant when an 
electrical charge is neutralized. Therefore, when the N/P ratio increased over 5, the RS peptide's hydrophobic interaction could help from more compact NCs, favorable for cell penetration. ${ }^{[29]}$

Second, hydrogen bonding of RS peptides could affect the NC formation and cell penetration. For example, in Fig. 2, the RS peptide formed more stable and compact NCs than the RT peptide. These compact and stable NCs could increase cell penetration efficiencies. First, RS peptide had more hydrogen bonding groups, including threonine (hydroxyl), glutamine (amino), tyrosine (hydroxyl), glutamic acid (carboxylate), than RT peptide's serine (hydroxyl) and threonine (hydroxyl). Therefore, RS peptides could form more hydrogen bonding with siRNAs, and hydrogen bonds could stabilize NCs. Second, hydroxyl, amino, carboxylic, and guanidinium groups of peptides were known to form hydrogen bonds with phosphate of nucleic acid or cellular membrane. ${ }^{[24,30-32]}$ Therefore, hydrogen bonding of RS peptides could increase interactions with membrane and cell-penetrating efficiencies. Also, hydrogen bonding and hydrophobicity of RS peptides increase the stability, improving cellular penetration efficiency. NC stability test indicated RS-NCs are more stable than $\mathrm{R}_{11^{-}}$or RT-NCs (Fig. S2, Table S3). The extended stability of RS-NCs also contributes to cell penetration.

The endocytosis pathways of RS-NC were revealed as lipid raft-mediated, clathrin-mediated endocytosis, micropinocytosis, and caveolin-mediated endocytosis in high order (Fig. 5). Generally, endocytosis pathways had a size range for effective working such as lipid raft-mediated endocytosis for particles less than $150 \mathrm{~nm}$, clathrin-mediated endocytosis for particles less than $200 \mathrm{~nm}$, micropinocytosis for particles over 1,000 nm, and caveolin-mediated endocytosis for particles less than $90 \mathrm{~nm} .{ }^{[33,34]}$ Thus, because the size distribution of RS-NCs ranged from $60 \mathrm{~nm}$ to 1,000 nm (Fig. S3), RS-NCs could be uptaken through four endocytosis pathways. Also, smaller NCs could become dissociated from big NCs. Moreover, the specificity of RS-NCs could be explained by the interaction between SP82 and specific surface receptors on breast cancer cells. ${ }^{[26,27]}$

Biosafety of $\mathrm{NC}$ was proved by maintaining the cell viability of RS peptide and that intradermally injected RS-NC did not induce tissue damage or inflammatory cell infiltration. Also, intradermally injected RS-NC did not raise TNF-alpha and IL-5 cytokine levels, causing inflammation. As a result, the RS-NC showed proper biocompatibility.

\section{Conclusion}

This study represented that newly designed RS fusion peptide could improve NC-mediated siRNA delivery targeting breast cancer cells. RS peptides could form self-assembled NCs with siRNAs via electrostatic attraction, hydrophobic interaction, and hydrogen bonding. Formed RS-NCs exhibited prolonged stability, enhanced cellular uptake, and target gene silencing by siRNAs. Resultantly, RS-NC-mediated siRNA delivery successfully inhibited breast cancer cell growth specifically and effectively. In addition, RS peptide was proved as cytocompatible, and RS-NC showed biosafety and no inflammation in vivo. Therefore, the RS-NC could be expected to become a promising siRNA delivery platform for breast cancer or other cancer treatment.

\section{Acknowledgment}

This research was supported by Basic Science Research Program through the National Research Foundation of Korea (NRF) funded by the Ministry of Science, ICT \& Future Planning (NRF-2021R1F1A104960811), and by the Incheon National University Research Grant in 2019.

\section{Conflict of interest}

The authors declare no financial or commercial conflict of interest.

\section{Data availability statement}

The data supporting the findings of this study are available from the corresponding author upon reasonable request.

\section{References}

1. Sung, H, Ferlay, J, Siegel, RL, Laversanne, M, Soerjomataram, I, Jemal, A, \& Bray, F. (2021). Global cancer statistics 2020: GLOBOCAN estimates of incidence and mortality worldwide for 36 cancers in 185 
countries. CA: A Cancer Journal for Clinicians, 71 (3), 209-249.

2. Nurgali, K.; Jagoe, R.T.; Abalo, R (2018). Editorial: Adverse effects of cancer chemotherapy: Anything new to improve tolerance and reduce sequelae? Frontiers in Pharmacology , 9 , 245.

3. Colleoni, M., \& Giobbie-Hurder, A. (2010). Benefits and adverse effects of endocrine therapy. Annals of Oncology , 21 (7), 107-111.

4. Pearce, A., Haas, M., Viney, R., Pearson, S. A., Haywood, P., Brown, C., \& Ward, R. (2017). Incidence and severity of self-reported chemotherapy side effects in routine care: A prospective cohort study.PLoS ONE, 12 (10), e0184360.

5. Berlanda, N., Somigliana, E., Frattaruolo, M. P., Buggio, L., Dridi, D., \& Vercellini, P. (2017). Surgery versus hormonal therapy for deep endometriosis: is it a choice of the physician? European Journal of Obstetrics and Gynecology and Reproductive Biology , 209 , 67-71.

6. Cross, D., \& Burmester, J. K. (2006). Gene Therapy for Cancer Treatment: Past, Present and Future. In Clinical Medicine 83 Research , 4 , 218-27.

7. Gardner, J. (1847). Potent and specific genetic interference by double-stranded RNA in Caenorhabditis elegans. The Lancet , 49 (1234), 431-434.

8. Ahlquist, P. (2002). RNA-Dependent RNA Polymerases, Viruses, and RNA Silencing. Science, 296, $1270-1273$.

9. Zaboikin, M., Srinivasakumar, N., \& Schuening, F. (2006). Gene therapy with drug resistance genes. Cancer Gene Therapy ,13 , 335-345.

10. Hoy, S. M. (2018). Patisiran: First Global Approval. Drugs ,78 (15), 1625-1631.

11. Scott, L. J. (2020). Givosiran: First Approval. Drugs ,80 (3), 335-339.

12. Garrelfs, S. F., Frishberg, Y., Hulton, S. A., Koren, M. J., O’Riordan, W. D., Cochat, P., Deschênes, G., Shasha-Lavsky, H., Saland, J. M., van't Hoff, W. G., Fuster, D. G., Magen, D., Moochhala, S. H., Schalk, G., Simkova, E., Groothoff, J. W., Sas, D. J., Meliambro, K. A., Lu, J., .. \& L Lieske, J. C. (2021). Lumasiran, an RNAi Therapeutic for Primary Hyperoxaluria Type 1. New England Journal of Medicine , 384 (13), 1216-1226.

13. Naik, S., Shreya, A. B., Raychaudhuri, R., Pandey, A., Lewis, S. A., Hazarika, M., Bhandary, S. V., Rao, B. S. S., \& Mutalik, S. (2021). Small interfering RNAs (siRNAs) based gene silencing strategies for the treatment of glaucoma: Recent advancements and future perspectives.Life Sciences , 264 , 118712.

14. Park, D., Yong Lee, J., Kyoung Cho, H., Jin Hong, W., Kim, J., Seo, H., Choi, I., Lee, Y., Kim, J., Min, S.-J., Yoon, S.-H., Sung Hwang, J., Jin Cho, K., \& Woong Kim, J. (2018). Cell-Penetrating Peptide-Patchy Deformable Polymeric Nanovehicles with Enhanced Cellular Uptake and Transdermal Delivery. Biomacromolecules, 19, 2682-2690 .

15. Rizzuti, M., Nizzardo, M., Zanetta, C., Ramirez, A., \& Corti, S. (2015). Therapeutic applications of the cell-penetrating HIV-1 Tat peptide. Drug Discovery Today , 20 (1), 76-85.

16. Yadav, D., Sandeep, K., Pandey, D., \& Dutta, R. K. (2017). Liposomes for Drug Delivery. Journal of Biotechnology \&3 Biomaterials, 7 (04), 276.

17. Lee, M. K. (2020). Liposomes for enhanced bioavailability of water-insoluble drugs: In vivo evidence and recent approaches.Pharmaceutics , 12 (3), 264.

18. Green, M., \& Loewenstein, P. M. (1988). Autonomous functional domains of chemically synthesized human immunodeficiency virus tat trans-activator protein. Cell , 55 (6), 1179-1188. 
19. Frankel, A. D., \& Pabo, C. O. (1988). Cellular uptake of the tat protein from human immunodeficiency virus. Cell , 55 (6), 1189-1193.

20. Guidotti, G., Brambilla, L., \& Rossi, D. (2017). Cell-Penetrating Peptides: From Basic Research to Clinics. Trends in Pharmacological Sciences , 38 (4), 406-424.

21. Jones, S. W., Christison, R., Bundell, K., Voyce, C. J., Brockbank, S. M. V, Newham, P., \& Lindsay, M. A. (2005). Characterisation of cell-penetrating peptide-mediated peptide delivery. British Journal of Pharmacology , 145, 1093-1102.

22. Park, J. W., Bang, E. K., Jeon, E. M., \& Kim, B. H. (2012). Complexation and conjugation approaches to evaluate siRNA delivery using cationic, hydrophobic and amphiphilic peptides. Organic and Biomolecular Chemistry , 10 (1), 96-102.

23. Oehlke, J., Scheller, A., Wiesner, B., Krause, E., Beyermann, M., Klauschenz, E., Melzig, M., \& Bienert, M. (1998). Cellular uptake of an $\alpha$-helical amphipathic model peptide with the potential to deliver polar compounds into the cell interior non-endocytically. Biochimica et Biophysica Acta - Biomembranes , 1414 (1-2), 127-139.

24. Walrant, A., Alves, I. D., \& Sagan, S. (2012). Molecular partners for interaction and cell internalization of cell-penetrating peptides : how identical are they ? Nanomedicine , 7 , 133-143.

25. Ryu, Y. C., Kim, K. A., Kim, B. C., Wang, H. M. D., \& Hwang, B. H. (2021). Novel fusion peptidemediated siRNA delivery using self-assembled nanocomplex. Journal of Nanobiotechnology ,19 (1), 1-18.

26. Sharma, S., Kotamraju, V. R., Mo, T., Tobi, A., Teesalu, T., \& Ruoslahti, E. (2013). Tumor-Penetrating Nanosystem Strongly Suppresses Breast Tumor Growth. Mol. Ther , $17,37$.

27. Lu, R.-M., Chen, M.-S., Chang, D.-K., Chiu, C.-Y., \& Lin, W.-C. (2013). Targeted Drug Delivery Systems Mediated by a Novel Peptide in Breast Cancer Therapy and Imaging. PLoS ONE , 8 (6), 66128.

28. Bourne, R. (2010). ImageJ. Fundamentals of Digital Imaging in Medicine , 9 (7), 185-188.

29. Rathnayake, P. V. G. M., Gunathunge, B. G. C. M., Wimalasiri, P. N., Karunaratne, D. N., \& Ranatunga, R. J. K. U. (2017). Trends in the binding of cell penetrating peptides to siRNA: A molecular docking study. Journal of Biophysics , 2017 (1), 1059216.

30. Mishra, A., Gordon, V. D., Yang, L., Coridan, R., \& Wong, G. C. L. (2008). HIV TAT forms pores in membranes by inducing saddle-splay curvature: Potential role of bidentate hydrogen bonding. Angewandte Chemie - International Edition , 47 (16), 2986-2989.

31. Rothbard, J. B., Jessop, T. C., \& Wender, P. A. (2005). Adaptive translocation: The role of hydrogen bonding and membrane potential in the uptake of guanidinium-rich transporters into cells. Advanced Drug Delivery Reviews , 57 (4), 495-504.

32. Loscalzo, D. E. H. R. C. J. (2011). Water-Protein Interactions of an Arginine-Rich Membrane Peptide in Lipid Bilayers Investigated by Solid-State NMR Spectroscopy. Bone , 23 (1), 1-7.

33. Kou, L., Sun, J., Zhai, Y., \& He, Z. (2013). The endocytosis and intracellular fate of nanomedicines: Implication for rational design. Asian Journal of Pharmaceutical Sciences , 8 (1), 1-10.

34. Manzanares, D., \& Ceña, V. (2020). Endocytosis: The nanoparticle and submicron nanocompounds gateway into the cell. Pharmaceutics ,12 (4), 1-22.

35. Bursa, F., Yellowlees, A., Bishop, A., Beckett, A., Hallis, B., \& Matheson, M. (2020). Estimation of ELISA results using a parallel curve analysis. Journal of Immunological Methods , $486,112836$.

Figure Legends 
Figure 1. The schematic image of novel fusion peptide design, NC formation with siRNAs, specific delivery, and inhibition of cell viability to breast cancer cells. The novel fusion peptide was designed as a combination of polyarginine, GGGG linker, and homing peptide. NCs $(278 \mathrm{~nm}, 10 \mathrm{mV})$ were self-assembled between peptides and siRNAs via electrostatic attraction and hydrogen bonds. NCs specifically entered into breast cancer cells through specific receptor clathrin-mediated endocytosis. CGI siRNAs repressed the breast cancer cell viability. The image was drawn using the Biorender software.

Figure 2. (A) Agarose gel electrophoresis images using gel retardation assay of RS- and RT-NCs. First, ten picomoles siRNAs were incubated with RS peptide (left) and RT peptide (right) at each N/P ratio for 30 minutes. Next, NCs were loaded into $1 \%$ agarose gel wells and electrophoresed at 100V for 35 minutes. The gel documentation system took gel images. (B) Hydrodynamic radius, polydispersity index, and zeta potential of RS-NC at each N/P ratio were measured by zetasizer $(\mathrm{n}=3)$. (C) Hydrodynamic radius, polydispersity index, and zeta potential of RT-NC at each N/P ratio were measured by zetasizer $(\mathrm{n}=3)$. (D) NC stability in Opti-MEM was assessed by a fluorescence cell counter. The number of three NCs was counted using ImageJ software. The NC count was normalized by the initial NC count.

Figure 3 . Flow cytometry results about cellular delivery of $\mathrm{R}_{11^{-}}$, RS- and RT-NCs with $200 \mathrm{nM}$ Cy3-labeled siRNAs. MDA-MB-231, MCF-7, BT-474, and SK-BR-3 were used as different types of breast cancer cells. RAW264.7 was used as a normal cell line. Red and green populations represented cell numbers of free siRNA and NC-mediated delivery, respectively. The number represented cell percentage with increased fluorescence compared to the control.

Figure 4. (A) Fluorescence imaging about cellular uptake of $\mathrm{R}_{11^{-}}$, RS- and RT-NCs with $200 \mathrm{nM}$ Cy3labeled siRNAs. Blue, green, and red fluorescences represented the nucleus (Hoechst 33342), actin filament (Phalloidin), and NCs (Cy3), respectively. The fluorescence cell image was observed using fluorescence microscopy at 150× magnification. Scale bar represented $100 \mu \mathrm{m}$ length. (B) Z-stack imaging about cellular internalization of RS-NC. Red and green fluorescence represented actin filament (Cy3) and RS-NC (FITC). Z-axis images were observed at $900 \times$ magnification. Scale bar represented $25 \mu \mathrm{m}$ length.

Figure 5. The endocytosis pathway is reduced by endocytosis inhibitors when RS-NC application. MDAMB-231 cells were incubated with each endocytosis inhibitor (Chlorpromazine, Methyl- $\beta$-cyclodextrin, Cytochalasin D, and Filipin III) for 30 minutes. Then, cells were incubated with RS NC with $200 \mathrm{nM}$ siRNA for four hours and analyzed using flow cytometry. Red and green populations represented cell counts without endocytosis inhibitor and with endocytosis inhibitor, respectively. The number represented the cell population with decreased fluorescence compared to the control.

Figure 6. (A) Reduced cell proliferation by siRNAs delivered via RS-NC at various N/P ratios. MDA-MB231 cells were incubated with RS-NC with 200 nM CGI siRNA for 24 hours and repeated once. (B) Reduced cell proliferation by $\mathrm{R}_{11}-\mathrm{NC}$ of various siRNA concentrations. MDA-MB-231 cells were incubated with $\mathrm{R}_{11}$-NC with various siRNA concentrations for 24 hours and repeated once. (C) Reduced cell proliferation by RS-NC of various siRNA concentrations. MDA-MB-231 cells were incubated with RS-NC with various siRNA concentrations for 24 hours and repeated once. Cell proliferation was measured using a CCK-8 assay kit. The Centerline and error bar represented mean and standard deviation normalized by the value of cell only. (D) Gene silencing by siRNA delivered via RS-NC. MDA-MB-231 cells were incubated with RS-NC with $200 \mathrm{nM}$ for anti-Kaiso siRNA for 24 hours. mRNA concentration was calculated by the $\Delta \Delta \mathrm{Ct}$ method using quantitative PCR of reversed transcribed cDNA. Centerline and error bar represented mean and standard deviation normalized by the absorbance at $450 \mathrm{nM}$ of the cell only condition. ${ }^{*},{ }^{* *}$, and ${ }^{* * *}$ mean p-value less than 0.05, 0.005, and 0.001 using T-test $(\mathrm{n}=12,2)$.

Figure 7. Cytocompatibility of RS and RT fusion peptides. (A) MDA-MB-231 cells and (B) HDFn cells were incubated with various concentrations of RS (left) and RT (right) peptides for 4 hours. Cell proliferation was measured using a CCK-8 assay kit. Centerline and error bar represented mean and standard deviation normalized by the absorbance at $450 \mathrm{nM}$ of the cell only condition. * means p-value less than 0.05 using T-test $(\mathrm{n}=8)$. 
Figure 8. (A) H\&E stained tissue observation of RS-NC-treated skin tissue. DPBS and RS-NC with 200 $\mathrm{nM}$ negative control siRNA were injected to epilated back of the Balb/c mouse, respectively. After one day, injected tissue was harvested, fixed, and stained by H\&E solution. Stained tissues were observed using a microscope at $20 \times$ magnification. Scale bar represented $100 \mu \mathrm{m}$ length. (B) TNF-alpha and IL-6 levels of RS-NC-treated skin tissue. Conditions of DPBS and RS-NC with $200 \mathrm{nM}$ negative control siRNA were injected to epilated back of the Balb/c mouse, respectively. After solubilization of harvested skin tissue, TNF-alpha (left) and IL-6 (right) levels were measured using each ELISA kit and 4 PL standard curve, respectively. The centerline and error bar represented mean and standard deviation. ${ }^{[35]}$
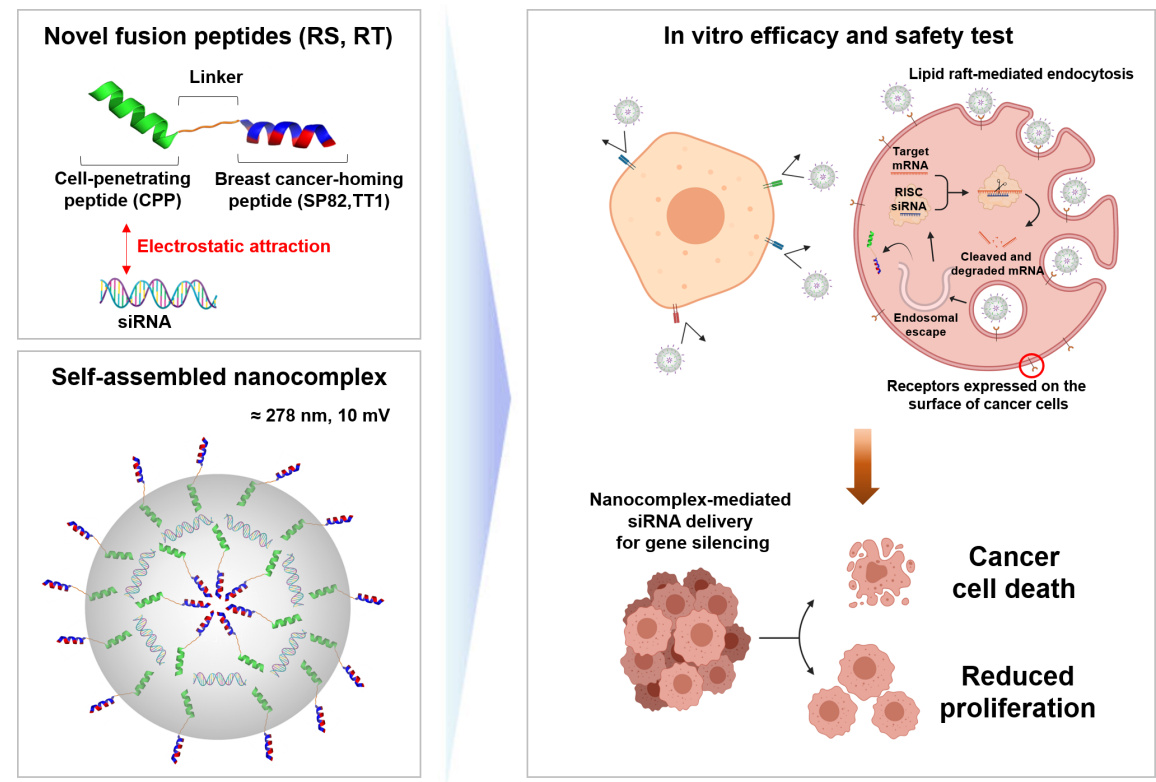

(A)
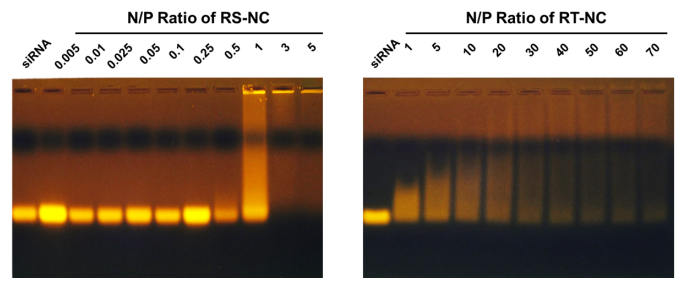

(D)

(B)
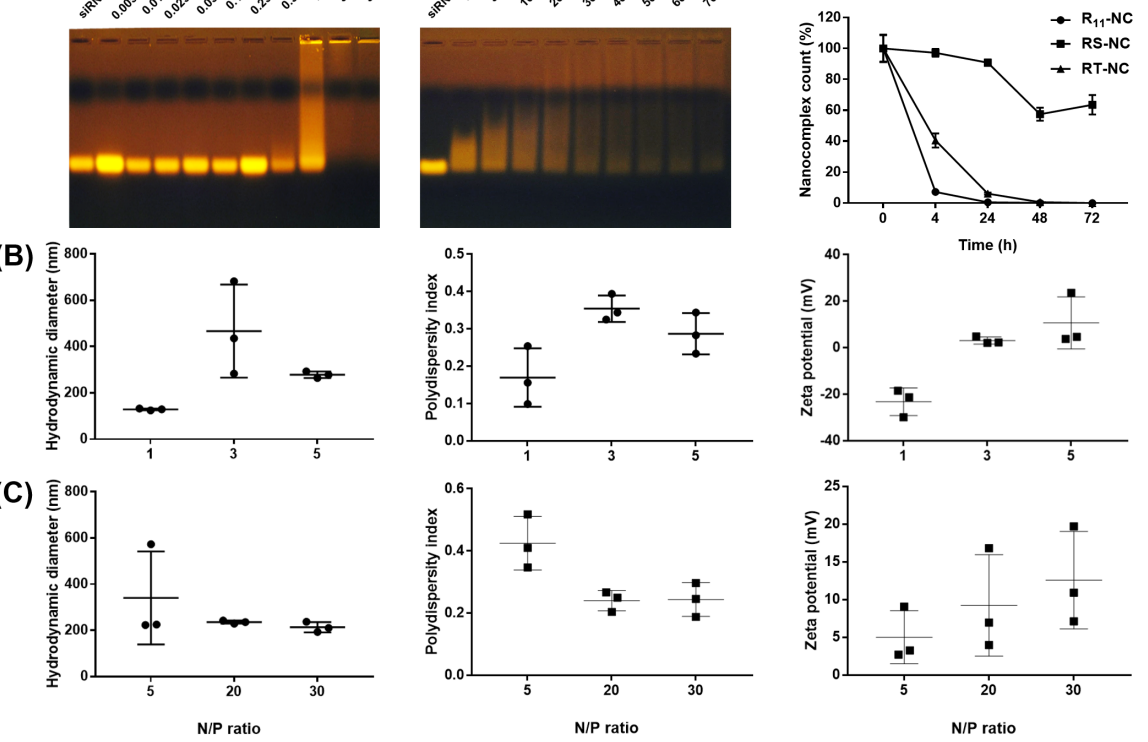

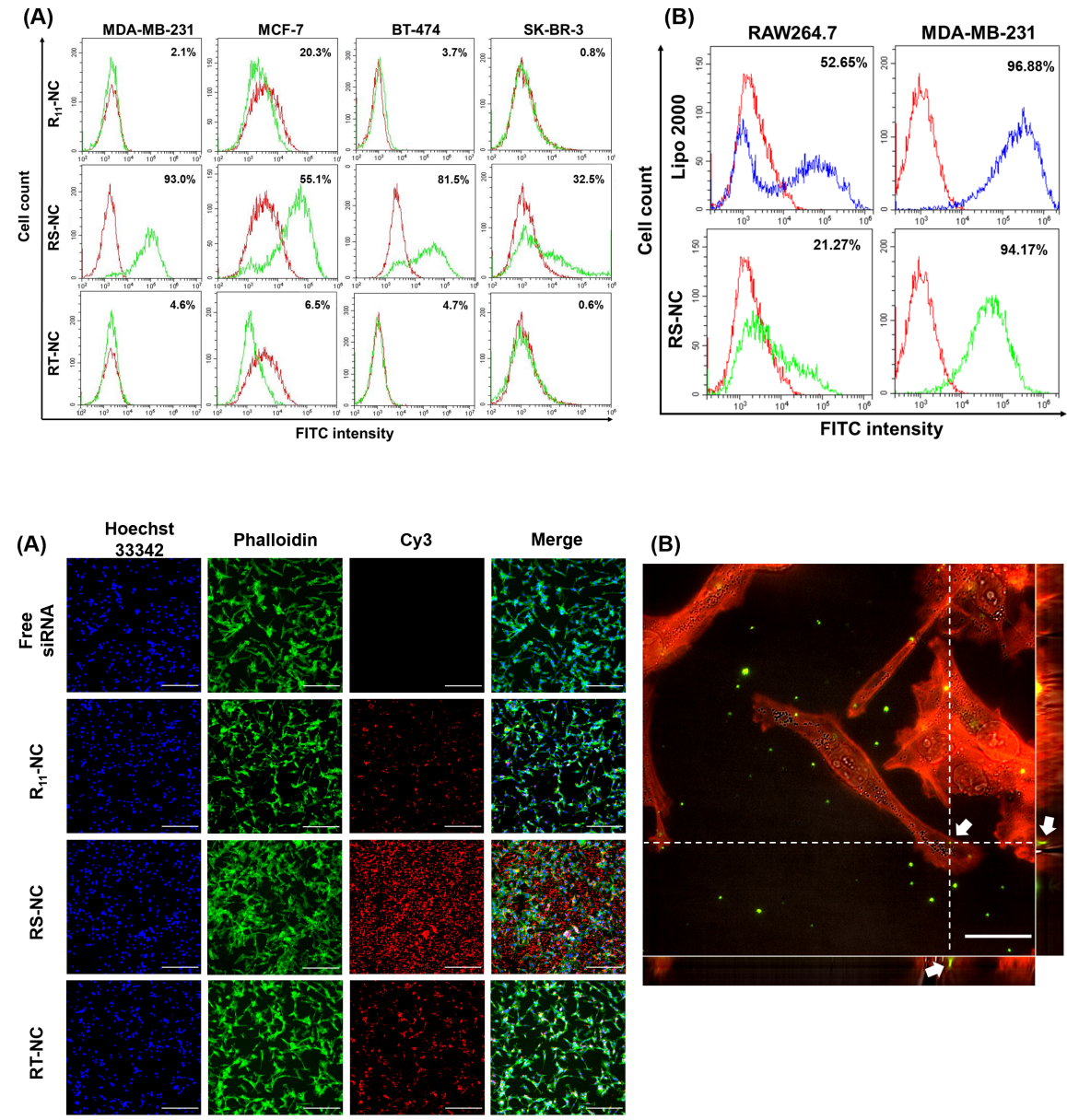

\section{(B)}
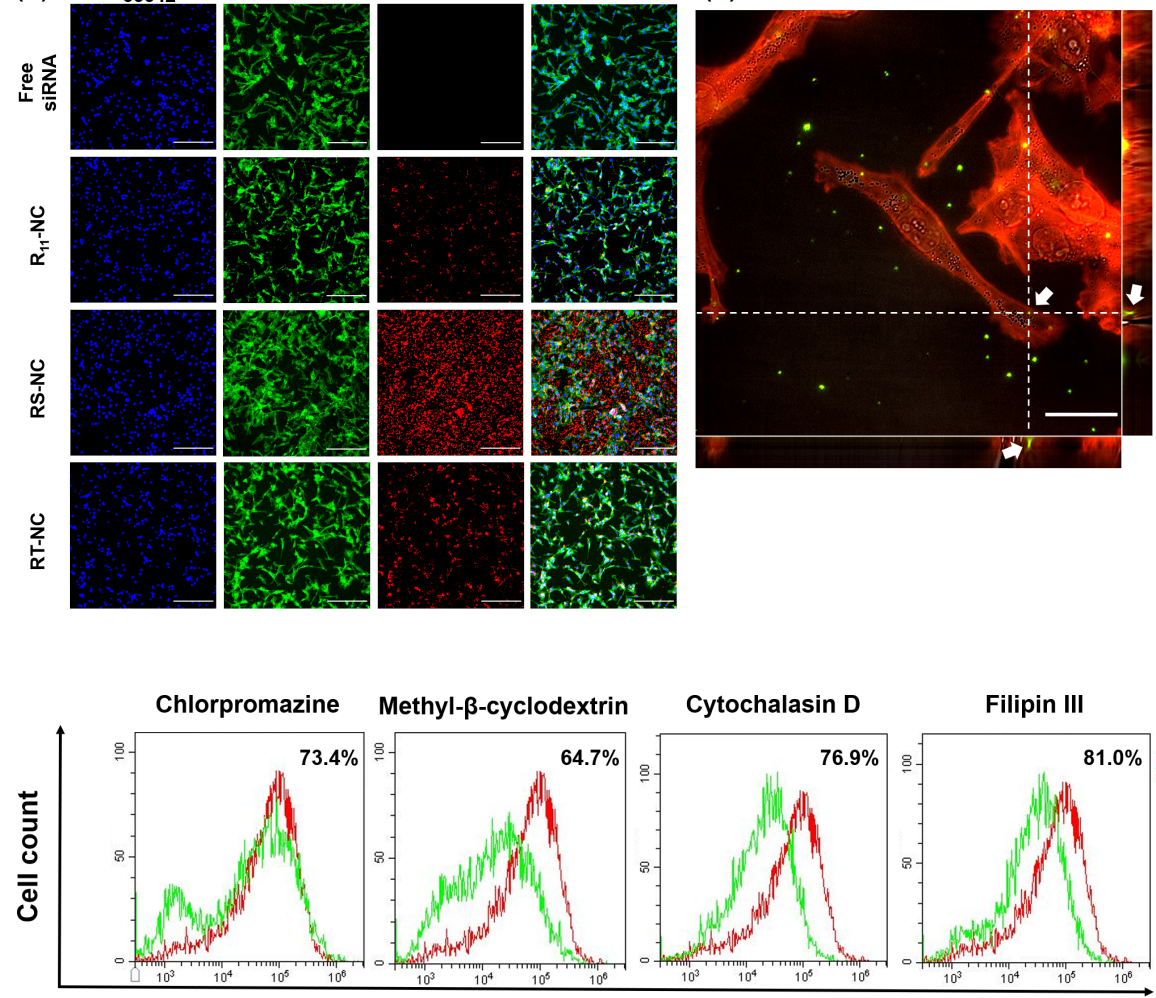

FITC intensity 
(A)

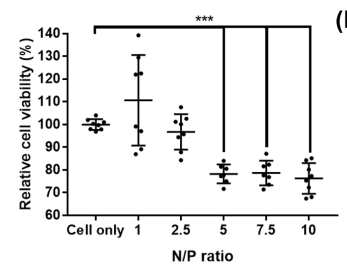

(D)

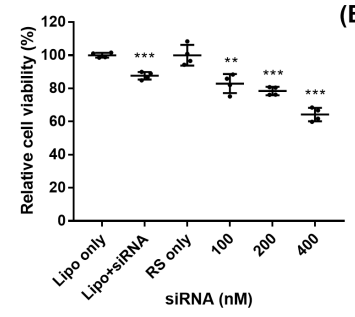

(B)

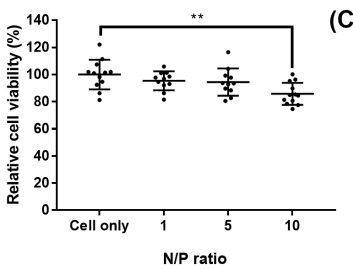

(E)

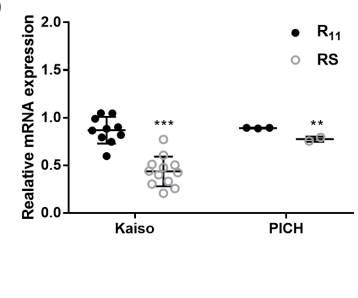

(C)

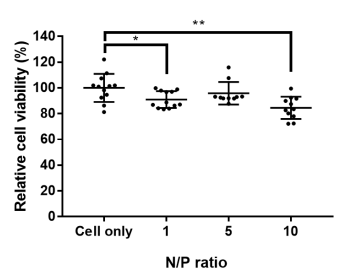

(A)
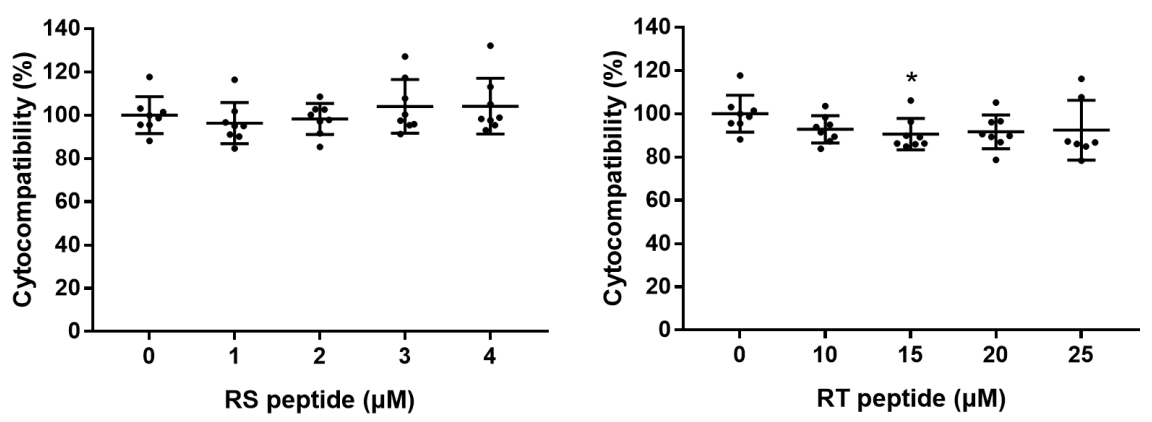

(B)
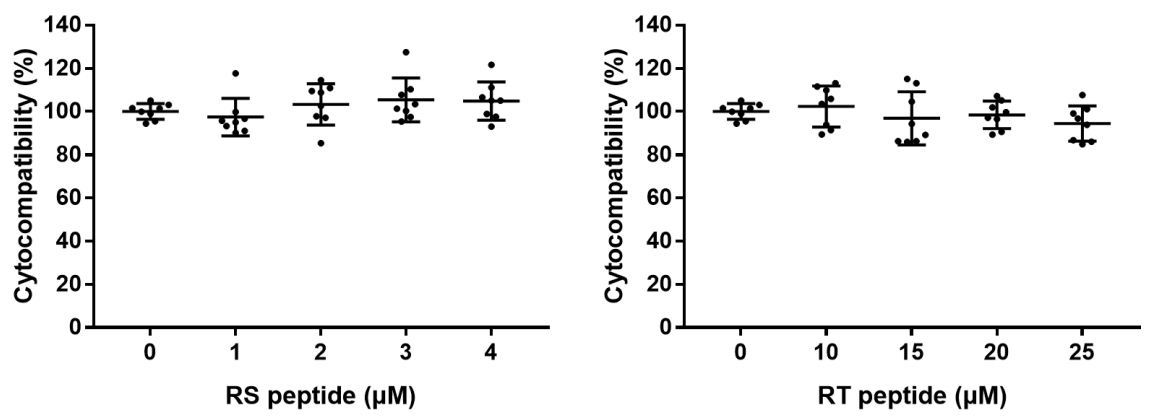
(A)

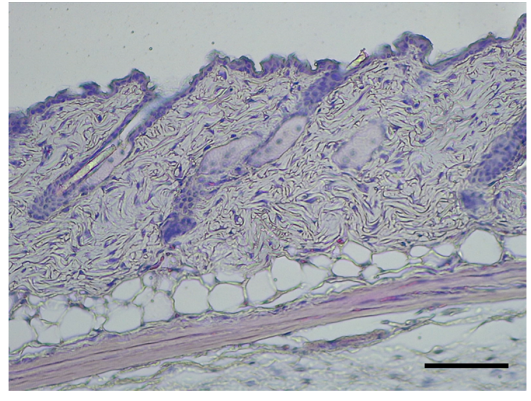

(B)

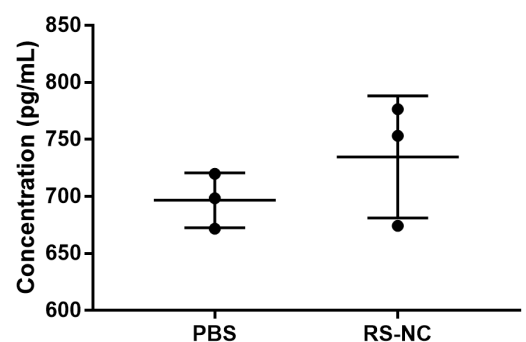

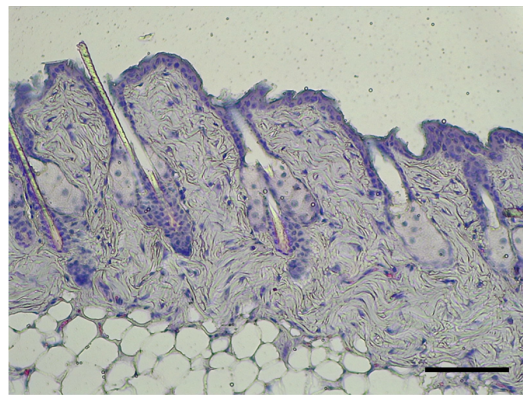

IL-6

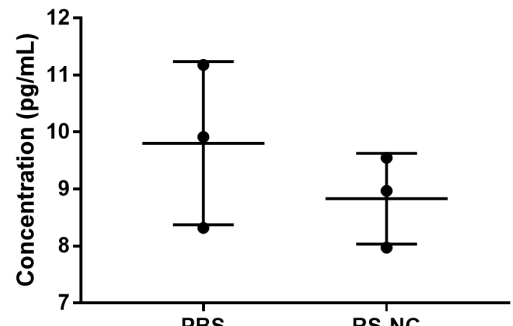

